\title{
Disentangling Highly Asymmetric Magnetoelectric Effects in Engineered Multiferroic Heterostructures
}

\author{
Enric Menéndez, ${ }^{1, *}$ Veronica Sireus, ${ }^{1}$ Alberto Quintana, ${ }^{1,2}$ Ignasi Fina, ${ }^{3}$ Blai Casals,${ }^{3}$ \\ Rafael Cichelero, ${ }^{3}$ Mikko Kataja, ${ }^{3}$ Massimiliano Stengel, ${ }^{3,4}$ Gervasi Herranz,${ }^{3}$ Gustau Catalán, $, 4,5$ \\ Maria Dolors Baró, ${ }^{1}$ Santiago Suriñach, ${ }^{1}$ and Jordi Sort ${ }^{1,4}$ \\ ${ }^{1}$ Departament de Física, Universitat Autònoma de Barcelona, E-08193 Bellaterra, Cerdanyola del Vallès, Spain \\ ${ }^{2}$ Department of Physics, Georgetown University, Washington DC, 20057, USA \\ ${ }^{3}$ Institut de Ciència de Materials de Barcelona (ICMAB-CSIC), Campus UAB, Bellaterra, E-08193 Barcelona, \\ Spain \\ ${ }^{4}$ Institució Catalana de Recerca i Estudis Avançats (ICREA), Pg. Lluís Companys 23, E-08010 Barcelona, Spain \\ ${ }^{5}$ Catalan Institute of Nanoscience and Nanotechnology (ICN2), CSIC and BIST, Campus UAB, Bellaterra, \\ E-08193 Barcelona, Spain
}

(Received 6 April 2019; revised manuscript received 30 May 2019; published 23 July 2019)

\begin{abstract}
One of the main strategies to control magnetism by voltage is the use of magnetostrictive-piezoelectric hybrid materials, such as ferromagnetic-ferroelectric heterostructures. When such heterostructures are subjected to an electric field, piezostrain-mediated effects, electronic charging, and voltage-driven oxygen migration (magnetoionics) may simultaneously occur, making the interpretation of the magnetoelectric effects not straightforward and often leading to misconceptions. Typically, the strain-mediated magnetoelectric response is symmetric with respect to the sign of the applied voltage because the induced strain (and variations in the magnetization) depends on the square of the ferroelectric polarization. Conversely, asymmetric responses can be obtained from electronic charging and voltage-driven oxygen migration. By engineering a ferromagnetic-ferroelectric hybrid consisting of a magnetically soft 50-nm thick $\mathrm{Fe}_{75} \mathrm{Al}_{25}$ (at. \%) thin film on top of a $\langle 110\rangle$-oriented $\mathrm{Pb}\left(\mathrm{Mg}_{1 / 3} \mathrm{Nb}_{2 / 3}\right) \mathrm{O}_{3}-32 \mathrm{PbTiO}_{3}$ ferroelectric crystal, a highly asymmetric magnetoelectric response is obtained and the aforementioned magnetoelectric effects can be disentangled. Specifically, the large thickness of the $\mathrm{Fe}_{75} \mathrm{Al}_{25}$ layer allows dismissing any possible charge accumulation effect, whereas no evidence of magnetoionics is observed experimentally, as expected from the high resistance to oxidation of $\mathrm{Fe}_{75} \mathrm{Al}_{25}$, leaving strain as the only mechanism to modulate the asymmetric magnetoelectric response. The origin of this asymmetric strain-induced magnetoelectric effect arises from the asymmetry of the polarization reversal in the particular crystallographic orientation of the ferroelectric substrate. These results are important to optimize the performance of artificial multiferroic heterostructures.
\end{abstract}

DOI: 10.1103/PhysRevApplied.12.014041

\section{INTRODUCTION}

Electric field control of magnetism has attracted much scientific and technological interest due to its energy efficiency implications, envisaging ultralow power dissipation magnetic storage and magnetoelectronic devices [1-3]. From a technological viewpoint, a reproducible, strong, and tunable magnetoelectric effect is required in a solid-state device configuration. So far, in contrast to single-phase multiferroics (with intrinsic magnetoelectric coupling) [4-6] and direct field effects (charge-mediated mechanism) in ultrathin metals and nanoporous alloys [7-10], the strain-mediated converse magnetoelectric

\footnotetext{
*enricmenendez@gmail.com
}

effect in magnetostrictive-piezoelectric hybrid materials (artificial multiferroics), such as ferromagnetic (FM)ferroelectric (FE) heterostructures, has resulted in one of the mechanisms with the largest possible voltage-driven modulation of the magnetic properties. Magnetostrictive FMs in combination with FE materials with strong piezoelectric properties are typically used [11-16]. Due to their high piezoelectric response, $(1-\mathrm{x}) \cdot \mathrm{Pb}\left(\mathrm{Mg}_{1 / 3}\right.$ $\left.\mathrm{Nb}_{2 / 3}\right) \mathrm{O}_{3}-\mathrm{x} \cdot \mathrm{PbTiO}_{3}(\mathrm{PMN}-\mathrm{x} \cdot \mathrm{PT})$ single crystals with compositions in the region close to the morphotropic phase boundary (i.e., $\mathrm{x}$ around $35 \mathrm{~mol} . \%$ ) are one of the most employed ferroelectric materials [17-22] in these engineered FM-FE heterostructures [13-15,23-33].

In general, when an artificial FM-FE heterostructure is subjected to an electric field, inverse magnetostriction in 
the FM induced by the piezoelectric strain arising from the FE, charge accumulation at the surface of the FM (typically only observed for thicknesses of a few nm) [7-10], and voltage-driven oxygen migration may simultaneously occur. This makes interpretation of the observed effects not straightforward, leading to misinterpretations. Moreover, when addressing the magnetoelectric response of a FM-FE heterostructure, not all the possible mechanisms are taken into account, resulting in another source of misunderstandings. Disentangling these diverse contributions is thus crucial for gaining further insight into the control of magnetism by voltage in FM-FE heterostructures and in the design and optimization of the next generation of magnetic storage and magnetoelectric devices.

In a first approximation, the electric-field modulation of magnetic properties via strain transfer in FMFE heterostructures should be symmetric with respect to voltage polarity (i.e., butterflylike magnetization voltage control). Additionally, since the strain largely vanishes after removal of the external electric field, the magnetic changes are generally volatile (i.e., not permanent) when the applied electric field is removed [15,30,31,34]. Furthermore, the magnetostrictive coupling is a function of the square of magnetization $(M)$ and electric polarization $(P)$ and, therefore, in principle, the sign of $M$ cannot be switched with $P$. However, strong asymmetries with voltage have been observed in the magnetic properties of FM-PMN-PT heterostructures, eventually resulting in a "looplike"-controlled magnetization, and therefore, in permanent changes (i.e., nonvolatile) [23-27]. Thus, if the magnitude of $P$ at $+\Delta V_{\text {applied }}$ is different from that at $-\Delta V_{\text {applied }}$, the magnitude of $M$ can be changed with voltage polarity. This asymmetric magnetoelectric effect is of potential interest for alternative types of energyefficient magnetic memories that would be solely controlled with electric voltage. Although the origin of this effect is often attributed to particular ferroelectric domain switching mechanisms of the FE, this nonvolatile behavior has also been linked to charge-mediated effects [28] or to a coexistence of strain- and charge-mediated magnetoelectric coupling (i.e., magnetoelectroelastic effects) due to the polarity-dependent nature of direct-field effects [29-31].

Moreover, in contrast to piezostrain and direct field effects, magnetoionics results not only in changes of extrinsic magnetic properties, such as coercivity or remanence, but also in saturation magnetization (which can precisely sense the degree of oxidation). In most of these studies, detailed investigations of eventual changes in the saturation magnetization with applied voltage have been largely omitted, thus overlooking possible voltage-driven oxygen diffusion (magnetoionics) phenomena [35-41], which also depend on the electric polarity. As a clear example, Ref. [40] reports on the voltage-controlled ON-OFF room temperature ferromagnetism in paramagnetic $\mathrm{Co}_{3} \mathrm{O}_{4}$. A negative voltage partly reduces $\mathrm{Co}_{3} \mathrm{O}_{4}$ to $\mathrm{Co}$ (ferromagnetism:
$\mathrm{ON}$ ), resulting in a graded material including Co- to Orich regions. A positive bias reverses the process oxidizing Co back to $\mathrm{Co}_{3} \mathrm{O}_{4}$ (ferromagnetism: OFF). Hence, full understanding of the asymmetric magnetoelectric effect in this type of multiferroic heterostructures (comprising PMN-PT) is lacking and deserves to be further studied in detail.

In this work, we disentangle the magnetoelectric effects of an engineered FM-FE heterostructure, which consists of a magnetically soft and isotropic 50-nm thick $\mathrm{Fe}_{75} \mathrm{Al}_{25}$ (at. \%) thin film grown on top of a $\langle 110\rangle$-oriented PMN32PT) ferroelectric crystal. The magnetoelectric properties are measured using vibrating sample magnetometry while applying out-of-plane electric fields and in-plane magnetic fields either along the [001] or [1-10] in-plane orthogonal directions of the substrate. Besides the low cost of $\mathrm{Fe}$ and $\mathrm{Al}, \mathrm{Fe}_{75} \mathrm{Al}_{25}$ alloys with large $\mathrm{Fe}$ contents are magnetostrictive [42-44], highly resistant to oxidation [45], and exhibit soft ferromagnetism [12]. This makes $\mathrm{Fe}_{75} \mathrm{Al}_{25}$ alloys a suitable candidate as FM in FM-FE hybrids. While the thickness of $50 \mathrm{~nm}$ disables any possible effect arising from charge accumulation, the high resistance to oxidation ensures minimized voltage-driven oxygen migration processes, leaving strain as the only mechanism to modulate the magnetoelectric response, which, in contrast to typical strain-induced magnetoelectric effects, is asymmetric with respect to the sign of the applied voltage. Depending on the direction of the applied magnetic field with respect to the in-plane crystallographic directions of the FE crystal (i.e., [001] and [1-10]), the degree of asymmetry of the straininduced magnetoelectric effects is different. Volatilelike (i.e., "butterflylike" and thus less asymmetric) magnetoelectric effects are observed when applying the magnetic field along [001], whereas a permanentlike (i.e., "looplike" and thus highly asymmetric) magnetoelectric response is distinguished along [1-10]. Anyhow, this asymmetric strain-induced magnetoelectric effect arises from the asymmetry of the polarization reversal in the ferroelectric substrate. [110]-oriented crystals are not isotropic in-plane (i.e., [001] and [1-10] orthogonal axes are not equivalent), and hence, the out-of-plane switching asymmetry of electric polarization translates via the in-plane anisotropy into an in-plane switching asymmetry in these crystals.

\section{EXPERIMENTAL DETAILS}

50-nm thick $\mathrm{Fe}_{75} \mathrm{Al}_{25}$ (at. \%) films are grown by cosputtering at room temperature on $\langle 110\rangle$-oriented PMN-32PT single crystals using an AJA International, Inc. magnetron sputtering system. Depositions are carried out at a rate of around $0.6 \AA / \mathrm{s}$.

The $\mathrm{Fe}_{75} \mathrm{Al}_{25}$ films are grown on $0.5-\mathrm{mm}$ thick and $3 \times 4 \mathrm{~mm}^{2}$ rectangular-shaped [110] PMN-PT substrates. The orthogonal in-plane directions of the substrates are 
determined by azimuthal XRD scans (not shown) laying the [001] and the [1-10] directions along the shortest and longest dimensions of the substrate, respectively. For simplicity, from now on, 0 and $90^{\circ}$ will refer to the magnetoelectric measurements carried out while applying the magnetic field along the [001] or [1-10] directions of the substrate, respectively.

SEM using secondary electrons in a FEI Magellan 400L microscope operated at $20 \mathrm{kV}$ is used to structurally and morphologically characterize the top surface of the samples. To investigate the inner parts of the as-grown samples, a cross-section lamella is prepared by focused ion beam and placed onto a $\mathrm{Cu}$ TEM grid. To characterize the cross section from structural and compositional viewpoints, TEM, energy dispersive X-ray (EDX) analysis, and high-angle annular dark-field scanning transmission electron microscopy (HAADF STEM) are performed using a TECNAI F20 HRTEM/STEM microscope operated at $200 \mathrm{kV}$. Further structural characterization is carried out by $\theta / 2 \theta$ XRD, grazing incidence XRD (GIXRD) with an incidence angle of $1^{\circ}$, and reciprocal space maps around (001) and (1-10) reflections of the PMN-32PT using $\mathrm{Cu} \mathrm{K} \mathrm{K}_{\alpha}$ radiation in a Malvern-PANalytical X'pert Pro materials research diffractometer.

The ferroelectric polarization versus applied voltage (i.e., electric field) loop is collected by grounding one of the electrodes (silver paste) while biasing the other (Fe-Al + silver paste) using the static hysteresis standard mode of the TFAnalyser2000 (Aixacct) with an integration time of $1 \mathrm{~ms}$ and a relaxation time of $1 \mathrm{~s}$. With this protocol, the displacive current flowing through the circuit $(I)$ is measured and the polarization is obtained from the integrated current in time and normalized by the area $(A): P=\frac{1}{A} \int I d t+C$. The constant $C$ is selected to obtain a loop where the polarization values at maximum negative and positive applied voltage are equal. The used sign convention is the standard, that is, the positive applied voltage corresponds applying to the PMN-PT electric field pointing toward the grounded electrode.

The magnetic and magnetoelectric properties are measured using a MicroSense (LOT-QuantumDesign) vibrating sample magnetometer (VSM), while the voltage is in situ applied out-of-plane using an Agilent B2902A power supply. A top electrode consisting of silver paint and the $\mathrm{Fe}_{75} \mathrm{Al}_{25}$ layer itself and a bottom silver paint electrode are used to apply the electric field across the FM-FE heterostructure. From an electric view point, the sample is out-of-plane poled using a silver paste bottom electrode, which sticks the sample to a nonconducting VSM holder and a top electrode made of silver paste and the conducting metallic $\mathrm{Fe}_{75} \mathrm{Al}_{25}$ layer.

The magnetoelectric characterization is carried out by in-plane vibrating sample magnetometry while out-ofplane poling (i.e., the electric field is applied along the [110] direction, which is perpendicular to the sample plane). A larger number of points are recorded close to the ferroelectric coercive field since it is in this region where the largest changes in strain are achieved.

\section{RESULTS AND DISCUSSION}

Substituting Fe by Al significantly increases the magnetostriction in $\mathrm{Fe}_{75} \mathrm{Al}_{25}$ alloys. However, this increase shows a maximal behavior for $\mathrm{Al}$ contents around 19 at. $\%$ [42]. At the same time, $\mathrm{Al}$ also plays a crucial role in enhancing the resistance to oxidation. The choice of a $\mathrm{Fe}_{75} \mathrm{Al}_{25}$ (at. \%) composition is aimed at having an alloy that simultaneously exhibits large magnetostriction [42-44] and high resistance to oxidation [45]. Even though lower $\mathrm{Al}$ contents down to 19 at. \% would result in a larger magnetostriction, oxidation resistance would then be worsened due to the limited amount of $\mathrm{Al}$.

By comparing the $\theta / 2 \theta$ XRD measurement of the PMN-32PT crystal and the $\mathrm{Fe}_{75} \mathrm{Al}_{25} / \mathrm{PMN}-32 \mathrm{PT}$ bilayer [Fig. 1(a)], it can be seen that there is only one peak corresponding to the $\mathrm{Fe}_{75} \mathrm{Al}_{25}$ film, located at around $44.4^{\circ}$. This is consistent with the (110) reflection of an $\mathrm{Fe}_{1-x} \mathrm{Al}_{\mathrm{x}}$ phase with $\mathrm{x} \approx 25$ at. \%, evidencing that the $\mathrm{Fe}_{1-\mathrm{x}} \mathrm{Al}_{\mathrm{x}}$ phase grows with a strong crystallographic texture. Nevertheless, the top view SEM image of the sample surface shown in Fig. 1(b) indicates that the sample is highly granular along the plane of the sample, showing a strong degree of in-plane polycrystallinity. Moreover, the (110) peak, which appears in the GIXRD measurement, evidences that the $\mathrm{Fe}_{1-\mathrm{x}} \mathrm{Al}_{\mathrm{x}}$ layer (Fig. S1), despite being highly textured, does not grow epitaxially. Figure 1(c) shows the TEM image of a cross section along the [1-10] direction of the heterostructure, evidencing that the $\mathrm{Fe}_{1-\mathrm{x}} \mathrm{Al}_{\mathrm{x}}$ layer is indeed around 50-nm thick. Moreover, the TEM images show that an interfacial layer between the substrate and the $\mathrm{Fe}_{1-\mathrm{x}} \mathrm{Al}_{\mathrm{x}}$ layer of about $5 \mathrm{~nm}$ is formed. The composition of the $\mathrm{Fe}_{75} \mathrm{Al}_{25}$ layer is determined to be $\mathrm{Fe}_{75} \mathrm{Al}_{25}$ (at. \%) by TEM-EDX. As can be seen in the compositional analysis carried out by HAADF STEM (Fig. S2), the interfacial layer is rich in $\mathrm{O}$ and Al. HRTEM [Fig. 1(d)] reveals that this interface layer is partly crystalline. The FFT corresponding to the red rectangle in Fig. 1(d) results in two spots, which correspond to an interplanar distance of $3.47 \AA$, which is compatible with the (012) planes of rhombohedral $\mathrm{Al}_{2} \mathrm{O}_{3}$ (00-001-1243), in concordance with the compositional mappings of Fig. S2.

The sample is sketched and the main crystallographic directions of the PMN-32PT crystal are represented in Fig. 2(a). Figure 2(b) shows the ferroelectric hysteresis loop measured in quasistatic conditions, whereas Fig. 2(c) is the square of the electric polarization represented in Fig. 2(b) as a function of electric field. The latter is proportional to the deformation that the PMN-PT undergoes when subjected to an electric field due to its piezoelectricity [46]. Interestingly, (110)-cut PMN-PT single crystals in 
(a)

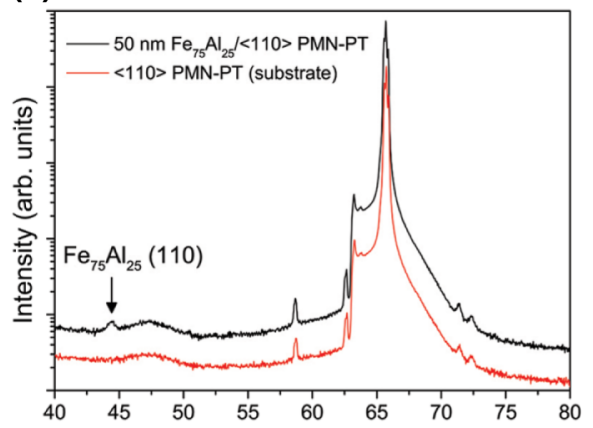

(c)

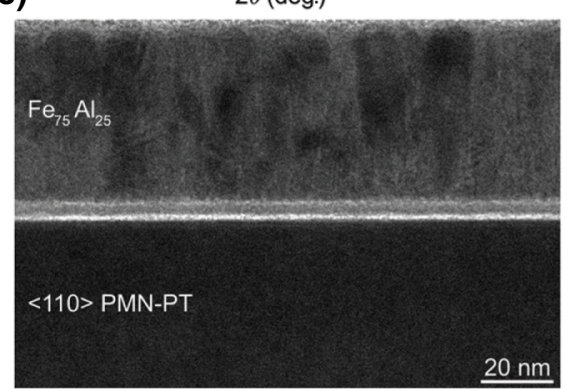

(b)

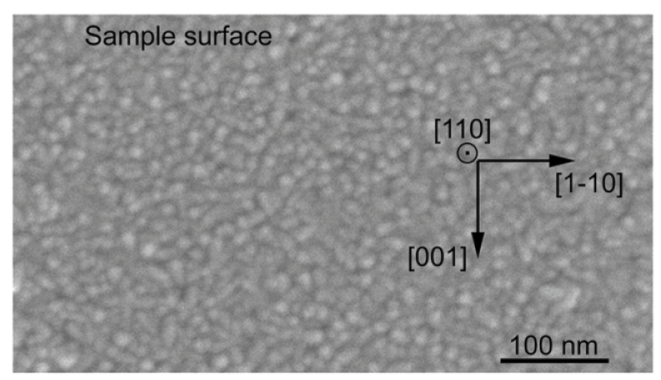

(d)

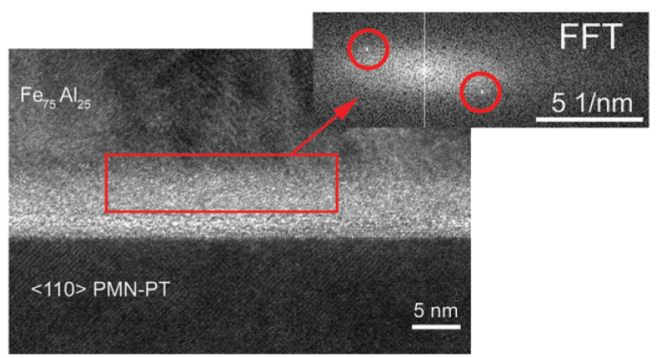

FIG. 1. (a) $\theta / 2 \theta$ x-ray diffraction patterns corresponding to a pristine (i.e., as-deposited) 50 -nm thick $\mathrm{Fe}_{75} \mathrm{Al}_{25} /\langle 110\rangle$ PMN-PT sample (black line) and a bare $\langle 110\rangle$ PMN-PT substrate (red line). (b) Scanning electron microcopy image taken using secondary electrons of the surface of a 50-nm thick $\mathrm{Fe}_{75} \mathrm{Al}_{25} /\langle 110\rangle$ PMN-PT sample. (c) TEM image of the cross section along [1-10] of a pristine 50-nm thick $\mathrm{Fe}_{75} \mathrm{Al}_{25} /\langle 110\rangle$ PMN-PT sample. (d) High resolution TEM image of the interface layer formed between the $\mathrm{Fe}_{75} \mathrm{Al}_{25}$ film and the PMN-PT substrate. The inset shows the FFT of the area marked with a red rectangle.

an out-of-plane poling configuration do not only display anisotropic in-plane piezoelectric coefficients, which results in compressive and tensile stresses along [001] and [1-10] directions, respectively, but also a strong strain response when $\mathrm{x}$ is within the region of the morphotropic phase boundary (i.e., $\mathrm{x}$ is around $35 \mathrm{~mol}$. \%) [18,46-48]. Remarkably, the ferroelectric loop of Fig. 2(b) is not symmetric. This could be ascribed to the interplay of the following aspects: (i) electrode asymmetry (the bottom electrode is made by silver paste, while the top electrode consists of the $\mathrm{Fe}_{75} \mathrm{Al}_{25}$ layer and silver paste), which might lead to asymmetric electric fields at the interfaces with the sample; (ii) macroscopic defect gradients [49]; and (iii) difficulty to reach saturation of the electric polarization (i.e., unsaturated loop), meaning that the initial polarity dominates thus, envisaging complex and path-dependent ferroelectric reversal mechanisms [50].

In the absence of an electric field, the sample shows an isotropic in-plane magnetic behavior (Fig. S3) as expected from the high degree of polycrystallinity in the plane of the sample [Fig. 1(b)]. This is very convenient to disentangle magnetoelectric effects since they may result in anisotropic changes of magnetic anisotropy as happens in other heterostructures, such as $\mathrm{Co}_{40} \mathrm{Fe}_{40} \mathrm{~B}_{20} / \mathrm{PMN}-\mathrm{PT}$ $[15,24,25]$.

Figure 3 shows the VSM characterization as a function of the applied voltage while applying the in-plane magnetic field along [001] [i.e., $0^{\circ}$ configuration, Fig. 2(a)], whereas Fig. 4 shows the magnetoelectric measurements when the in-plane magnetic field is applied along [1-10] (i.e., $90^{\circ}$ configuration). Specifically, the samples are subjected to the following electric protocol (see "Voltage protocol" in the Supplemental Material [51]): First, hysteresis loops are recorded while following the ascending branch $(A)$ of the ferroelectric loop. The piezoelectric is always brought to ferroelectric saturation at $-6 \mathrm{kV} / \mathrm{cm}$ before applying the indicated measurement voltage to achieve better control of the sample state. Then, the protocol is reversed (i.e., from positive to negative saturation) to apply electric fields corresponding to the descending branch $(D)$ of the ferroelectric loop.

For the $0^{\circ}$ configuration, although the behavior is asymmetric, the dependences of both the remanence (i.e., squareness, $\left.m_{R} / m_{S}\right)$ and the coercivity $\left(H_{C}\right)$ with the applied electric field along [001] show an important component with a butterflylike shape [Figs. 3(b) and 3(c), respectively], resembling the variation of strain with voltage in a piezoelectric material [Fig. 2(c)]. This is consistent with the converse magnetoelectric effect being dominated by strain coupling via inverse magnetostriction. In addition to the strain contribution, the observed asymmetry with electric field polarity could be a fingerprint of the coexistence of strain and charge effects through magnetoelectroelastic effects [30], since strain is proportional 
(a)

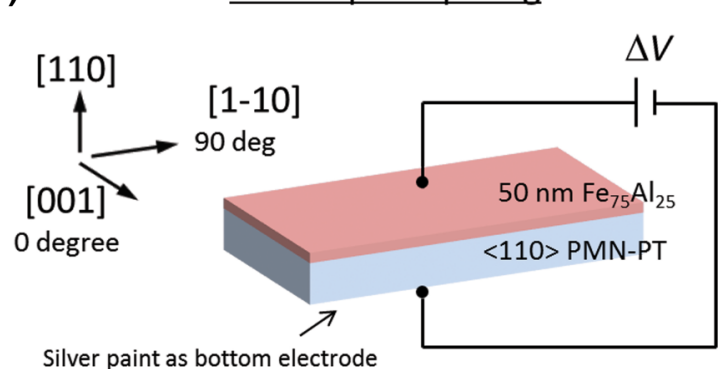

(b)

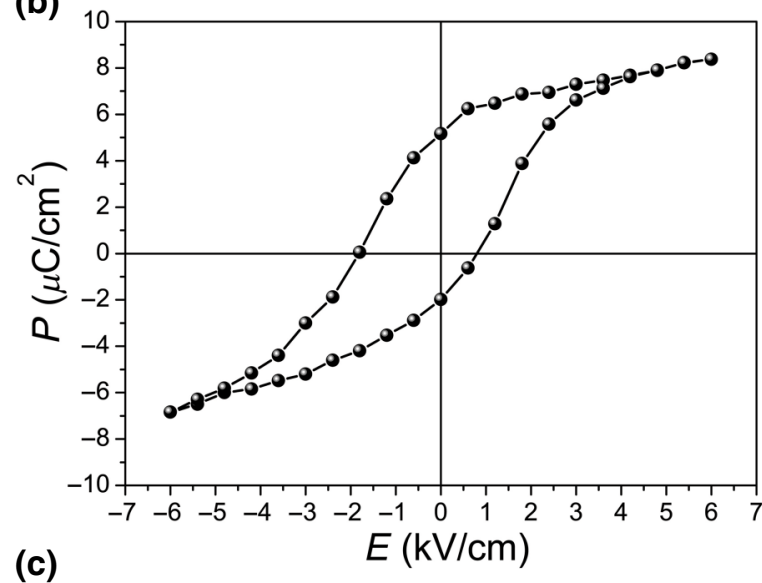

(c)

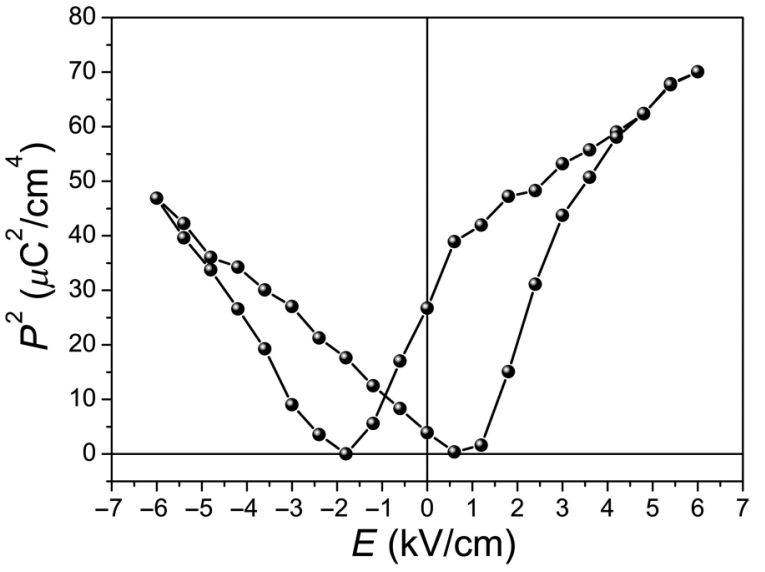

FIG. 2. (a) Schematic representation of the out-of-plane poling applied to the samples. (b) Ferroelectric hysteresis loop (electric polarization $P$ vs applied electric field $E$ ) of an as-deposited 50-nm thick $\mathrm{Fe}_{75} \mathrm{Al}_{25} /\langle 110\rangle$ PMN-PT sample. (c) Square of $P$ (which is proportional to the piezoelectric strain) vs $E$.

to $P^{2}$ whereas direct field effects are a function of $P$. However, taking into account that the electric field in metals (e.g., $\mathrm{Fe}_{75} \mathrm{Al}_{25}$ ) is strongly screened at the surface, resulting in no penetration beyond the Thomas-Fermi screening length (which is around $0.5 \mathrm{~nm}$ ) [9,52], charge effects due to the accumulation of electrostatic charges at the surface are rather unlikely to be significant since the $\mathrm{Fe}_{75} \mathrm{Al}_{25}$ layers are 50 -nm thick. Anyhow, this inplane magnetic asymmetry could be linked to the nonsymmetric perpendicular-to-plane ferroelectric behavior of
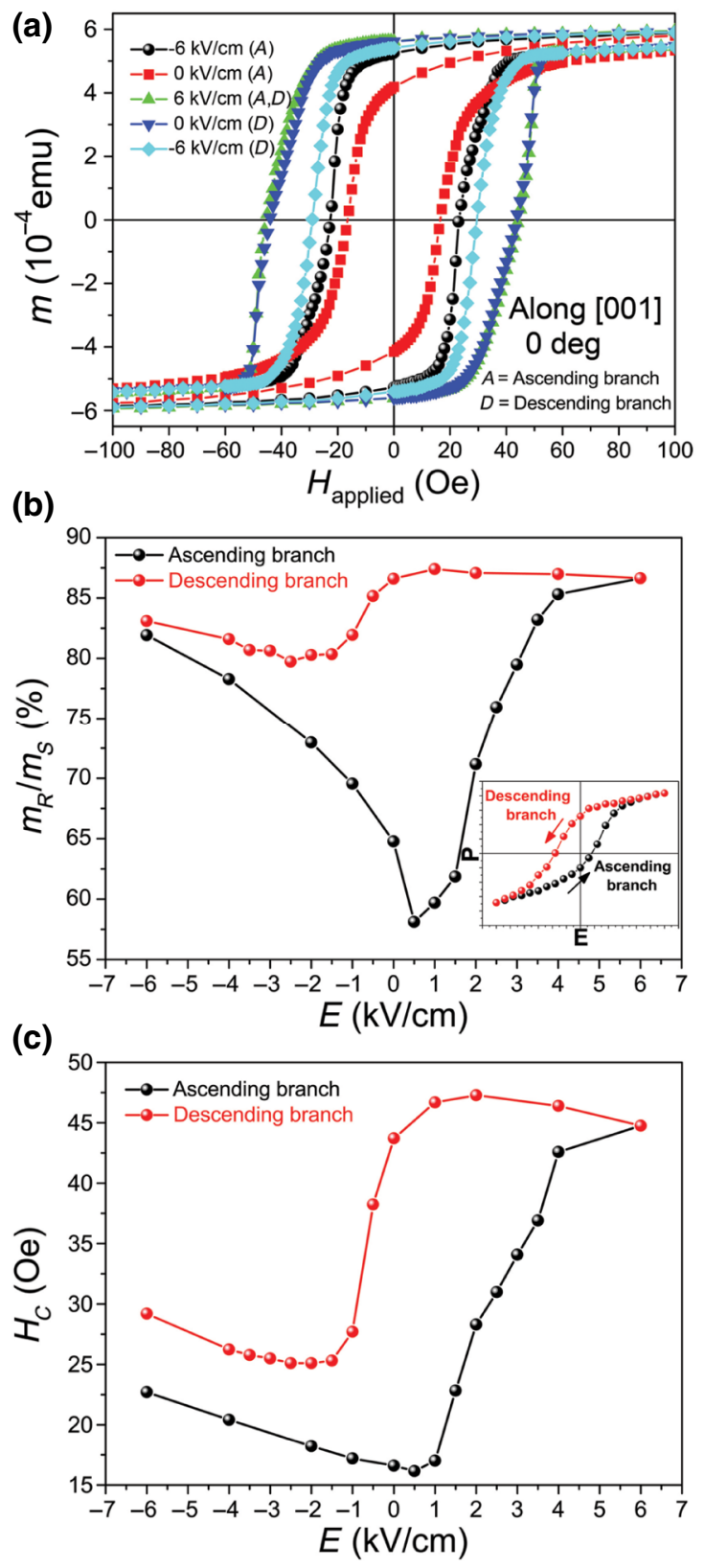

FIG. 3. (a) Hysteresis loops recorded along in-plane direction [001] ( $0^{\circ}$ configuration) by vibrating sample magnetometry at a given applied electric field applied out-of-plane. The piezoelectric is always brought to ferroelectric saturation at $-6 \mathrm{kV} / \mathrm{cm}$ before registering the magnetic measurement. (b) and (c) dependences of the magnetic moment at remanence normalized by the magnetic moment at saturation $\left(m_{R} / m_{S}\right)$ and coercivity $\left(H_{C}\right)$ with the applied electric field. Ascending $(A)$ and descending $(D)$ refer to the branches of the ferroelectric hysteresis loop as sketched in the inset of panel (b).

the PMN-PT [i.e., nonsaturated (i.e., minor) ferroelectric loop], envisaging that path-dependent mechanisms of electric polarization reversal could also be a source of magnetic asymmetry. 
(a)

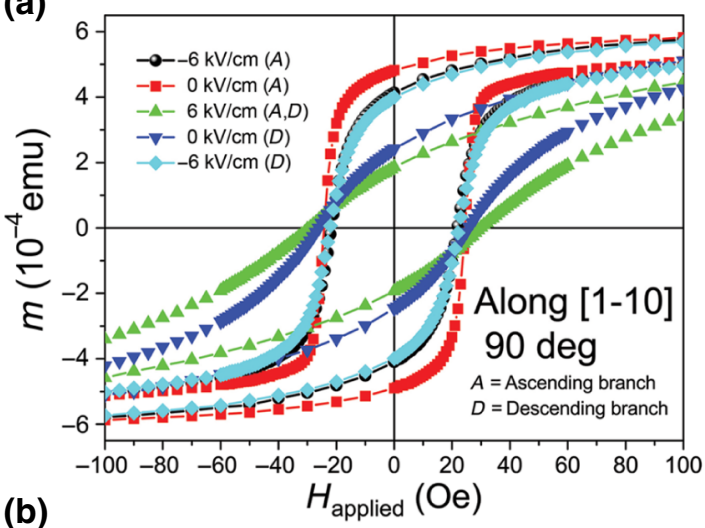

(b)

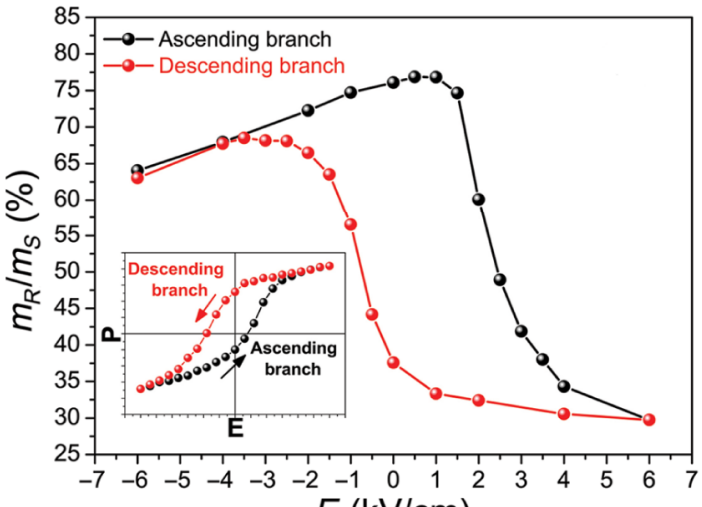

(c)

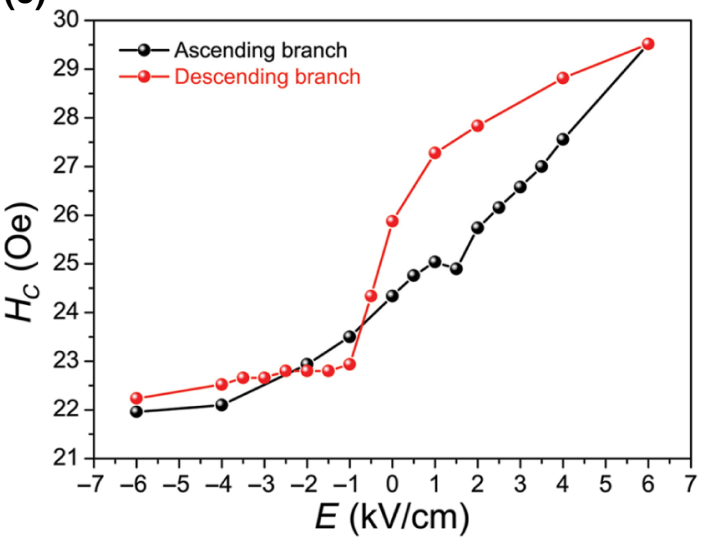

FIG. 4. (a) Hysteresis loops recorded along in-plane direction [1-10] ( $90^{\circ}$ configuration) by vibrating sample magnetometry at a given applied electric field out-of-plane. The piezoelectric is always brought to ferroelectric saturation at $-6 \mathrm{kV} / \mathrm{cm}$ before registering the magnetic measurement. (b) and (c) dependences of the magnetic moment at remanence normalized by the magnetic moment at saturation $\left(m_{R} / m_{S}\right)$ and coercivity $\left(H_{C}\right)$ with the applied electric field. Ascending $(A)$ and descending $(D)$ refer to the branches of the ferroelectric hysteresis loop as sketched in the inset of panel (b).

A quite different dependence of the magnetoelectric coupling to the polarity of the electric field is observed when the magnetization is measured along the [1-10] direction (i.e., $90^{\circ}$ configuration). In this case, while the dependence of the magnetic coercivity on the applied electric field is rather weak, that of the squareness (i.e., $m_{R} / m_{S}$ ) exhibits a looplike shape with an asymmetry with respect to the electric polarity much more pronounced than when magnetization is measured along the [001] direction. Remarkably, from negative to positive electric fields, the magnetic properties switch from an easy to a hard axis behavior. As possible origins of this observation, electronic charging, oxygen migration, and strain-mediated effects will be discussed in the following.

First, although the $\mathrm{Fe}_{75} \mathrm{Al}_{25}$ film is exceedingly thick to present significant charge-mediated effects, the effects of charge accumulation might propagate within the spinspin exchange correlation length, which extends several $\mathrm{nm}$ toward the interior of the alloy [52]. To verify this possibility, a thinner $\mathrm{Fe}_{75} \mathrm{Al}_{25}$ film (20-nm thick) is grown in the same fashion with the aim to enhance eventual surface charging effects. As can be seen in Fig. S4, the dependence of the squareness (i.e., $m_{R} / m_{S}$ ), along [1-10] (i.e., $90^{\circ}$ configuration), with the applied electric field also exhibit a looplike dependence but the relative changes are, in fact, less pronounced in this thinner sample. Specifically, for the $50-\mathrm{nm}$ thick sample, the maximum relative change in remanence is around $61.4 \%$, whereas, for the $20-\mathrm{nm}$ thick sample, the change is $53.6 \%$, indicating that interfacial charge effects (and also their propagation via the spin-spin correlation length) are ruled out as the main mechanism governing this reorientation of the magnetic easy axis with voltage. This sample also shows an $\mathrm{Al}_{2} \mathrm{O}_{3}$-based interfacial layer of around $5 \mathrm{~nm}$ in thickness at the interface with the PMN-PT. Regarding the role of these $\mathrm{Al}_{2} \mathrm{O}_{3}$-based interfacial layers in possible charge-mediated effects, 50$\mathrm{nm}$ and 20 -nm thick $\mathrm{Fe}_{75} \mathrm{Al}_{25}$ layers are still too thick to exhibit significant direct field effects. Effectively, since $\mathrm{Al}_{2} \mathrm{O}_{3}$ is also highly insulating, the interfacial layers add up to the PMN-PT, resulting in a slightly thicker insulating substrate.

After discarding charge effects, we focus on possible voltage-driven oxygen migration (i.e., magnetoionics) phenomena since this magnetoelectric mechanism is polarity dependent and it could result in strong permanent (nonvolatile) changes [35-38,40,41]. As can be seen in Fig. 5, the magnetic moment at saturation remains virtually constant during the voltage protocol (changes are lower than $2 \%$ and the maximum change is related to an increase in magnetic moment), indicating that no oxidation occurs during voltage actuation. This in agreement with the structural characterization, which evidences the formation of an interfacial layer based on $\mathrm{Al}_{2} \mathrm{O}_{3}$, which acts as a barrier for oxygen migration, isolating and protecting the system from further oxidation. The $\theta / 2 \theta$ XRD measurement of a sample subjected to a full voltage protocol shows no traces of phases other than $\mathrm{Fe}_{75} \mathrm{Al}_{25}$, further confirming the protective character of the $\mathrm{Al}_{2} \mathrm{O}_{3}$-based interface layer (Fig. S5) and the resistance of $\mathrm{Fe}_{75} \mathrm{Al}_{25}$ to oxidation. 

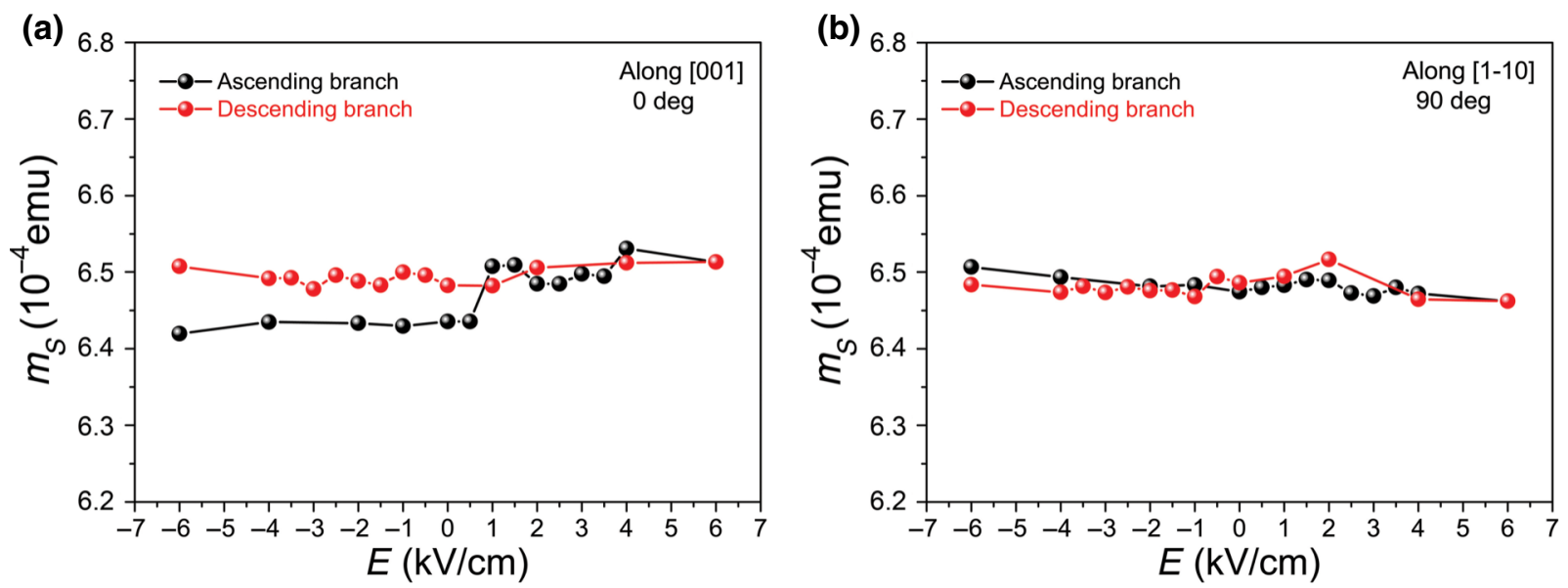

FIG. 5. (a) and (b) dependences of the magnetic moment at saturation with the applied electric field when the magnetic field is applied along the [001] ( $0^{\circ}$ configuration) or [1-10] (90 configuration) directions, respectively.

Taking into account the asymmetry in the measured ferroelectric hysteresis loop of the PMN-PT [Fig. 2(b)] and ruling out voltage-driven electronic charging and oxygen migration effects, the magnetoelectric behavior of the sample seems to be solely governed by strain-mediated effects (i.e., by the ferroelectric and/or piezoelectric nature of the PMN-PT). Thus, the strong in-plane magnetoelectric asymmetry along in-plane [001] and [1-10] axes in our [110]-oriented crystals might be related to the specific symmetry related ferroelectric response of our [110]oriented PMN-PT crystals as compared to the more usual [100]-oriented substrates. PMN-PT compositions around the morphotropic phase boundary exhibit a complex phase diagram [19]. Specifically, poling along [110] results in [111] and [11-1] as statistically equivalent spontaneous polarization directions [21]. Moreover, considering a pseudocubic cell for [110] PMN-PT, [111] fully falls in the plane determined by [110] and [001] (Fig. S6). Ferroelectric reversal in this material can take place via nucleation and domain wall movements of $71^{\circ}, 109^{\circ}$, and $180^{\circ}$ domain walls [53-56]. $\mathrm{In} \mathrm{BiFeO}_{3}$, which is a model multiferroic with a rather similar crystalline structure, the domain wall energy $\gamma$ follows the sequence: $\gamma_{109^{\circ}}<\gamma_{180^{\circ}}<\gamma_{71^{\circ}}$ [54]. Specifically, on top of single $180^{\circ}$ switching, there are consecutive $71^{\circ}+109^{\circ}$ switching events. Domain walls of $180^{\circ}$ and $71^{\circ}$ imply a polarization reversal, which takes place in the same plane (i.e., the plane where [111] lies), resulting in minimized inplane distortions along [1-10]. Conversely, $109^{\circ}$ domain walls lead to a $90^{\circ}$ twist of the polarization (the polarization rotates out of the plane of the domain wall), giving rise to significant in-plane distortions along [1-10]. To some extent, $109^{\circ}$ domain walls resemble ferromagnetic Bloch domain walls [55]. In other words, particularly when loops are unsaturated, a path-dependent polarization reversal, on top of $180^{\circ}$ switching events, is enabled through ferroelastic switching (consecutive $71^{\circ}+109^{\circ}$ switching). In crystals oriented with [001] out of the plane (and thus parallel to the electric field), this occurs isotropically: all in-plane switching directions are energetically equivalent, thus all ferroelastic switching events are equally probable and happen in the same proportion. In contrast, [110]oriented crystals are not isotropic in-plane (i.e., [001] and [1-10] orthogonal axes are not equivalent), and hence, the out-of-plane switching asymmetry of electric polarization [Figs. 2(b) and 2(c)] translates, via the in-plane anisotropy, into an in-plane switching asymmetry in these crystals.

In order to investigate the ferroelectric reversal of the PMN-PT, we perform in-plane reciprocal space mappings around the (001) and (1-10) x-ray diffraction reflections of a PMN-PT substrate in pristine conditions (i.e., $0 \mathrm{~V}$ in Fig. 6) and at $0 \mathrm{~V}$ after applying $-6 \mathrm{kV} / \mathrm{cm}$ (i.e., remanence from $-6 \mathrm{kV} / \mathrm{cm}$ in Fig. 6). The (001) and (1-10) XRD reflections of [110] PMN-PT correspond to the inplane orthogonal [001] and [1-10] directions, respectively. This allows us to investigate the influence of the electric field on the interplanar distances $d_{001}$ and $d_{1-10}$ and thus on the electric field-induced deformation along the shortest and longest dimensions of the sample. To estimate these interplanar distances, the maximum intensity of the peaks is considered. Prior to the voltage application, the interplanar distances are $d_{001}=4.02385 \AA\left(q_{x^{\prime}}=0.24852 \AA^{-1}\right)$ and $d_{1-10}=2.84725 \AA\left(q_{y^{\prime}}=0.35122 \AA^{-1}\right)$. Upon voltage actuation at negative ferroelectric remanence, $d_{001}=$ $4.02375 \AA\left(q_{x^{\prime}}=0.24852 \AA^{-1}\right)$ and $d_{1-10}=2.84275 \AA$ $\left(q_{y^{\prime}}=0.35177 \AA^{-1}\right)$. In absolute values, along [001], the interplanar distance remains almost unaltered $(0.0025 \%$ of change), whereas along [1-10], there is a permanent change of around $0.16 \%$ of the initial value. Interestingly, it is along this direction where the magnetic properties exhibit a pronounced nonvolatile behavior (looplike dependence). In contrast to the $71^{\circ}$ and $180^{\circ}$ domains, 

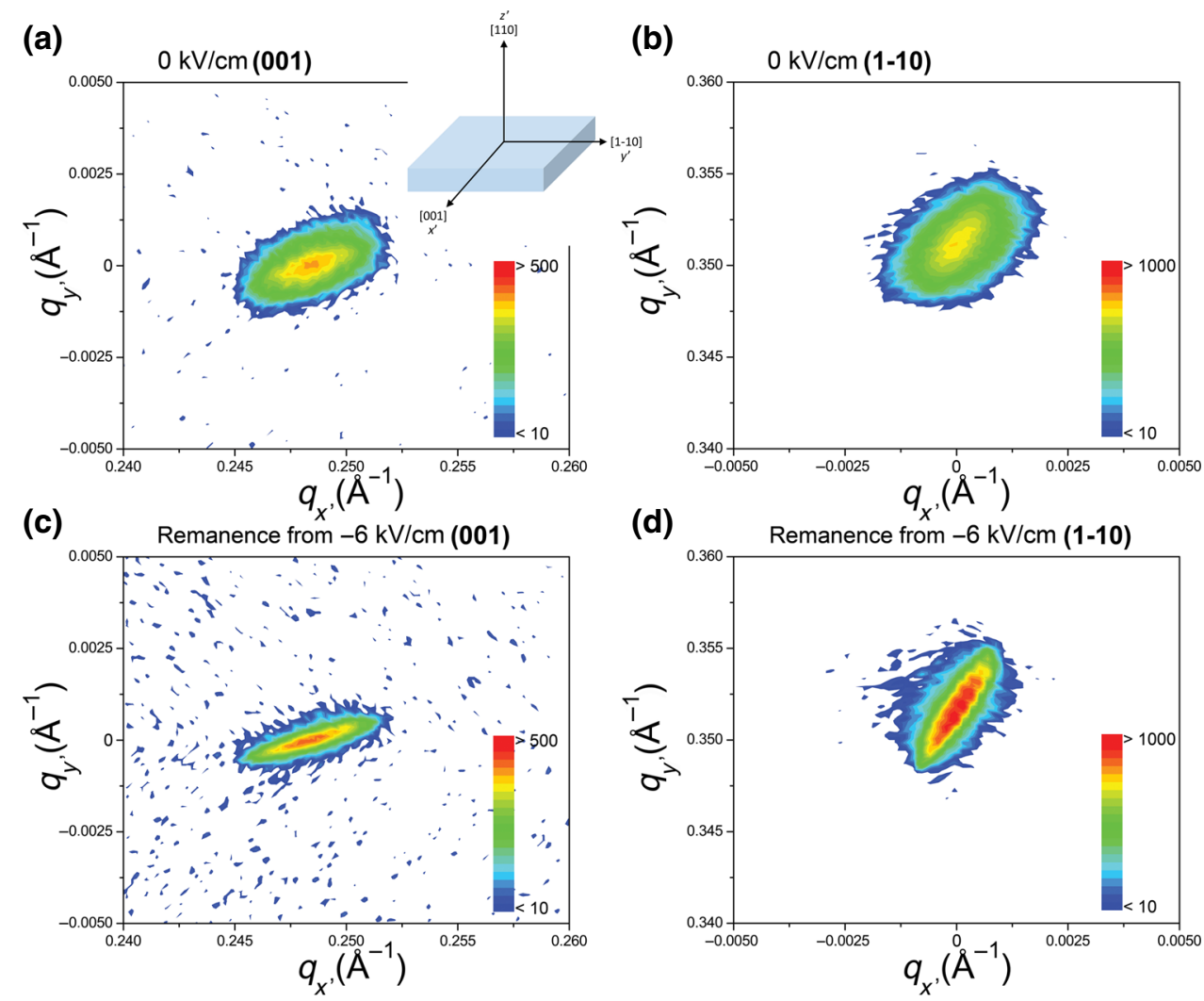

FIG. 6. (a) and (b) in-plane reciprocal space mappings around the (001) and (1-10) planes of a pristine substrate (i.e., $0 \mathrm{kV} / \mathrm{cm})$, respectively. (c) and (d) in-plane reciprocal space mappings around the (001) and (1-10) planes of a PMN-PT substrate at negative ferroelectric remanence (measured at $0 \mathrm{kV} / \mathrm{cm}$ after being subjected to $-6 \mathrm{kV} / \mathrm{cm}$ ), respectively.

the electric polarization reversal through the $109^{\circ}$ domains takes place via a $90^{\circ}$ twist when reversing the electric field, which results in additional stress-induced anisotropy along [1-10], thus explaining the strong asymmetry with voltage polarity of the magnetic properties in this direction and their nonvolatility upon removing the electric voltage. As can also be seen in Fig. 6, electric field actuation results in sharpened peaks. This could be ascribed to a decrease in mosaicity in the plane of the sample due to the undergone deformation. Concerning the role of the $\mathrm{Al}_{2} \mathrm{O}_{3}$-based interfacial layers in these piezostrain-mediated effects, the strain transfer from PMN-PT to the $\mathrm{Fe}_{75} \mathrm{Al}_{25}$ layer should not be significantly weakened by the $\mathrm{Al}_{2} \mathrm{O}_{3}$-based interfacial layer because, as can be seen in Fig. 1(d), this layer is very thin and it grows rather epitaxially with the PMN-PT.

\section{CONCLUSIONS}

The magnetoelectric behavior of 50-nm thick $\mathrm{Fe}_{75} \mathrm{Al}_{25}$ (at. \%) films grown onto [110] PMN-32PT substrates is investigated in detail by performing in-plane vibrating sample magnetometry along the [001] and [1-10] directions of the PMN-PT while applying an external out-of-plane electric field. The magnetic moment at saturation remains virtually unaltered with voltage, ruling out voltage-driven $\mathrm{O}$ migration as an operative magnetoelectric mechanism. Conversely, both the remanence (i.e., $\left.m_{R} / m_{S}\right)$ and the coercivity $\left(H_{C}\right)$ exhibit a strong dependence on the applied voltage (amplitude and sign). In perfect crystals with symmetric electrodes such asymmetry should not exist. However, crystals are never perfectly symmetric [49], and the ferroelectric hysteresis measurements (Fig. 2) show that the positive and negative polarities are nonequivalent in our substrates. Likewise, along [001], $m_{R} / m_{S}$ and $H_{C}$ show a slightly asymmetric butterflylike dependence, while along [1-10], $m_{R} / m_{S}$ exhibits a clear looplike behavior, evidencing strong nonvolatile properties. Asymmetry can sometimes be attributed to interfacial charging effects. However, electrostatic charge effects are mostly significant when dealing with ultrathin films and a thinner $\mathrm{Fe}_{75} \mathrm{Al}_{25}$ sample grown in the same fashion as a thicker one exhibits reduced permanent effects, so interfacial charging is ruled out as the explanation. Thus, the combination of macroscopic crystal asymmetry plus strain is left as the most plausible mechanism to modulate the magnetoelectric response of the investigated system. Indeed, detailed structural characterization by reciprocal space mappings around the (001) and (1-10) $\mathrm{x}$-ray diffraction reflections of a PMN-PT substrate reveal that the application of an electric field results in permanent 
strains only in the [1-10] direction. This explains the strong nonvolatility of the magnetic properties, which is linked to ferroelastic switching (consecutive $71^{\circ}+109^{\circ}$ switching) rather than single $180^{\circ}$ switching events. $109^{\circ}$ domain switching implies a $90^{\circ}$ twist of the polarization when reversing polarity, thus causing a stress-induced magnetic anisotropy along [1-10], explaining the dissimilar magnetic behavior with voltage polarity along the direction, which shows irreversible and permanent strain upon removing the electric voltage. Our results reveal that, in order to properly understand magnetoelectric mechanisms in artificial FM-FE heterostructures, it is necessary to clearly assess the intrinsic polarization reversal of the ferroelectric material, especially when using PMN-PT or other complex ferroelectric materials in these artificial multiferroic materials. Finally, even though the sign of magnetization cannot be switched with electric polarization, the magnitude of magnetization can be changed with voltage polarity (i.e., different magnitudes of electric polarization between plus and minus $\Delta V_{\text {applied }}$ ), implying that strain-mediated effects could eventually be used for memory purposes.

\section{ACKNOWLEDGMENTS}

Financial support by the European Research Council (SPIN-PORICS 2014-Consolidator Grant, Agreement No. 648454), the Spanish Government (Grants No. MAT2017-86357-C3-1-R, No. MAT2017-85232-R (AEI/FEDER,UE), and No. MAT2015-73839-JIN, and associated FEDER), the Generalitat de Catalunya (Grants No. 2017-SGR-292 and No. 2017301 SGR1377) and the European Union's Horizon 2020 research and innovation programme under the Marie Skłodowska-Curie Grant No. 665919 is acknowledged. I. F. acknowledges his "Ramón y Cajal" Grant No. RYC-2017-22531. The ICN2 is funded by the CERCA programme/Generalitat de Catalunya. ICN2 and ICMAB also acknowledge financial support from the Spanish Ministry of Economy, Competitiveness and Universities through the "Severo Ochoa" Programme for Centres of Excellence in R\&D (Grants No. SEV-2017-0706 and No. SEV-2015-0496, respectively).

[1] C. Song, B. Cui, F. Li, X. Zhou, and F. Pan, Recent progress in voltage control of magnetism: materials, mechanisms, and performance, Prog. Mater. Sci. 87, 33 (2017).

[2] J.-M. Hu, Z. Li, L.-Q. Chen, and C.-W. Nan, Highdensity magnetoresistive random access memory operating at ultralow voltage at room temperature, Nat. Commun. 2 , 553 (2011).

[3] Y. Shiota, T. Nozaki, F. Bonell, S. Murakami, T. Shinjo, and Y. Suzuki, Induction of coherent magnetization switching in a few atomic layers of FeCo using voltage pulses, Nat. Mater. 11, 39 (2012).
[4] W. Eerenstein, N. D. Mathur, and J. F. Scott, Multiferroic and magnetoelectric materials, Nature 442, 759 (2006).

[5] G. Catalan and J. F. Scott, Physics and applications of Bismuth ferrite, Adv. Mater. 21, 2463 (2009).

[6] N. Izyumskaya, Y. Aliyov, and H. Morkoç, Oxides, oxides, and more oxides: high-( oxides, ferroelectrics, ferromagnetics, and multiferroics, Crit. Rev. Solid State Mater. Sci. 34, 89 (2009).

[7] M. Weisheit, S. Fähler, A. Marty, Y. Souche, C. Poinsignon, and D. Givord, Electric field-induced modification of magnetism in thin-film ferromagnets, Science 315, 349 (2007).

[8] C.-G. Duan, J. P. Velev, R. F. Sabirianov, Z. Zhu, J. Chu, S. S. Jaswal, and E. Y. Tsymbal, Surface magnetoelectric effect in ferromagnetic metal films, Phys. Rev. Lett. 101, 137201 (2008).

[9] I. V. Ovchinnikov and K. L. Wang, Theory of electric-fieldcontrolled surface ferromagnetic transition in metals, Phys. Rev. B 79, 020402(R) (2009).

[10] A. Quintana, J. Zhang, E. Isarain-Chávez, E. Menéndez, R. Cuadrado, R. Robles, M. D. Baró, M. Guerrero, S. Pané, B. J. Nelson, C. M. Müller, P. Ordejón, J. Nogués, E. Pellicer, and J. Sort, Voltage-induced coercivity reduction in nanoporous alloy films: a boost toward energy-efficient magnetic actuation, Adv. Funct. Mater. 27, 1701904 (2017).

[11] Y. Wang, J. Hu, Y. Lin, and C.-W. Nan, Multiferroic magnetoelectric composite nanostructures, NPG Asia Mater. 2, 61 (2010).

[12] Y. Wei, C. Gao, Z. Chen, S. Xi, W. Shao, P. Zhang, G. Chen, and J. Li, Four-state memory based on a giant and non-volatile converse magnetoelectric effect in FeAl/PINPMN-PT structure, Sci. Rep. 6, 30002 (2016).

[13] M. Liu, O. Obi, J. Lou, Y. Chen, Z. Cai, S. Stoute, M. Espanol, M. Lew, X. Situ, K. S. Ziemer, V. G. Harris, and N. X. Sun, Giant electric field tuning of magnetic properties in multiferroic ferrite/ferroelectric heterostructures, Adv. Mater. 19, 1826 (2009).

[14] T. Wu, A. Bur, K. Wong, P. Zhao, C. S. Lynch, P. K. Amiri, K. L. Wang, and G. P. Carman, Giant electric-field-induced reversible and permanent magnetization reorientation on magnetoelectric $\mathrm{Ni} /(011)$ $\left[\mathrm{Pb}\left(\mathrm{Mg}_{1 / 3} \mathrm{Nb}_{2 / 3}\right) \mathrm{O} 3\right]_{(1-\mathrm{x})}-\left[\mathrm{PbTiO}_{3}\right]_{\mathrm{x}}$ heterostructure, Appl. Phys. Lett. 98, 012504 (2011).

[15] S. Zhang, Y. Zhao, X. Xiao, Y. Wu, S. Rizwan, L. Yang, P. Li, J. Wang, M. Zhu, H. Zhang, X. Jin, and $\mathrm{X}$. Han, Giant electrical modulation of magnetization in $\mathrm{Co}_{40} \mathrm{Fe}_{40} \mathrm{~B}_{20} / \mathrm{Pb}\left(\mathrm{Mg}_{1 / 3} \mathrm{Nb}_{2 / 3}\right)_{0.7} \mathrm{Ti}_{0.3} \mathrm{O}_{3}$ heterostructure, Sci. Rep. 4, 3727 (2014).

[16] T. H. E. Lahtinen, K. J. A. Franke, and S. van Dijken, Electric-field control of magnetic domain wall motion and local magnetization reversal, Sci. Rep. 2, 258 (2012).

[17] S. W. Choi, T. R. Shrout, S. J. Jang, and A. S. Bhalla, Dielectric and pyroelectric properties in the $\mathrm{Pb}\left(\mathrm{Mg}_{1 / 3} \mathrm{Nb}_{2 / 3}\right) \mathrm{O}_{3}-\mathrm{PbTiO}_{3}$ system, Phys. Rev B 100, 29 (1989).

[18] S.-E. Park and T. R. Shrout, Ultrahigh strain and piezoelectric behavior in relaxor based ferroelectric single crystals, J. Appl. Phys. 82, 1804 (1997). 
[19] B. Noheda, D. E. Cox, G. Shirane, J. Gao, and Z.-G. Ye, Phase diagram of the ferroelectric relaxor $(1-\mathrm{x}) \cdot \mathrm{Pb}\left(\mathrm{Mg}_{1 / 3} \mathrm{Nb}_{2 / 3}\right) \mathrm{O}_{3}-\mathrm{x} \cdot \mathrm{PbTiO}_{3}$, Phys. Rev. B 66, 054104 (2002).

[20] T. Wu, P. Zhao, M. Bao, A. Bur, J. L. Hockel, K. Wong, K. P. Mohanchandra, C. S. Lynch, and G. P. Carman, Domain engineered switchable strain states in ferroelectric $(011)[\mathrm{Pb}(\mathrm{Mg} 1 / 3 \mathrm{Nb} 2 / 3) \mathrm{O} 3]_{(1-\mathrm{x})}$-[PbTiO3 $]_{\mathrm{x}}(\mathrm{PMN}-$ $\mathrm{PT}, \mathrm{x} \approx 0.32$ ) single crystals, J. Appl. Phys. 109, 124101 (2011).

[21] G. Sébald, L. Lebrun, and D. Guyomar, Stability of morphotropic $<110>$ oriented $0.65 \mathrm{PMN}-0.35 \mathrm{PT}$ single crystals, Phys. Rev B 51, 1491 (2004).

[22] P. Kumar, C. Prakash, and T. C. Goel, Dielectric and electrostrictive properties of PMNT near MPB, Sci. Technol. Adv. Mater. 8, 463 (2007).

[23] T. Wu, A. Bur, K. Wong, P. Zhao, C. S. Lynch, P. K. Amiri, K. L. Wang, and G. P. Carman, Electrical control of reversible and permanent magnetization reorientation for magnetoelectric memory devices, Appl. Phys. Lett. 98, 262504 (2011).

[24] S. Zhang, et al., Electric-Field Control of Nonvolatile Magnetization in $\mathrm{Co}_{40} \mathrm{Fe}_{40} \mathrm{~B}_{20} / \mathrm{Pb}\left(\mathrm{Mg}_{1 / 3} \mathrm{Nb}_{2 / 3}\right)_{0.7} \mathrm{Ti}_{0.3} \mathrm{O}_{3}$ Structure at Room Temperature, Phys. Rev. Lett. 108, 137203 (2012).

[25] P. Li, Y. Zhao, S. Zhang, A. Chen, D. Li, J. Ma, Y. Liu, D. T. Pierce, J. Unguris, H.-G. Piao, H. Zhang, M. Zhu, X. Zhang, X. Han, M. Pan, and C.-W. Nan, Spatially resolved ferroelectric domain-switching-controlled magnetism in $\mathrm{Co}_{40} \mathrm{Fe}_{40} \mathrm{~B}_{20} / \mathrm{Pb}\left(\mathrm{Mg}_{1 / 3} \mathrm{Nb}_{2 / 3}\right)_{0.7} \mathrm{Ti}_{0.3} \mathrm{O}_{3}$ multiferroic heterostructure, ACS Appl. Mater. Interfaces 9, 2642 (2017).

[26] X. Han, Y. Li, X. Guo, Z. Wang, Y. Zuo, L. Xi, and D. Xue, Large electric tunable remanent magnetization and in-plane anisotropy field in (110) $\mathrm{PMN}-0.32 \mathrm{PT} / \mathrm{Cu} / \mathrm{Fe}_{65} \mathrm{Co}_{35}$ multiferroic heterostructures, Mater. Res. Express 1, 026103 (2014).

[27] L. Yang, Y. Zhao, S. Zhang, P. Li, Y. Gao, Y. Yang, H. Huang, P. Miao, Y. Liu, A. Chen, C. W. Nan, and C. Gao, Bipolar loop-like non-volatile strain in the (001)-oriented $\mathrm{Pb}\left(\mathrm{Mg}_{1 / 3} \mathrm{Nb}_{2 / 3}\right) \mathrm{O}_{3}-\mathrm{PbTiO}_{3}$ single crystals, Sci. Rep. 4, 4591 (2014).

[28] C. Zhang, F. Wang, C. Dong, C. Gao, C. Jia, C. Jiang, and D. Xue, Electric field mediated non-volatile tuning magnetism at the single-crystalline $\mathrm{Fe} / \mathrm{Pb}\left(\mathrm{Mg}_{1 / 3} \mathrm{Nb}_{2 / 3}\right)_{0.7}$ $\mathrm{Ti}_{0.3} \mathrm{O}_{3}$ interface, Nanoscale 7, 4187 (2015).

[29] Y. T. Yang, J. Li, X. L. Peng, B. Hong, X. Q. Wang, H. L. Ge, D. H. Wang, and Y. W. Du, Surface-effect enhanced magneto-electric coupling in FePt/PMN-PT multiferroic heterostructures, AIP Adv. 7, 055833 (2017).

[30] J. Heider, M. Fechner, R. V. Chopdekar, C. Piamonteze, J. Dreiser, C. A. Jenkins, E. Arenholz, S. Rusponi, H. Brune, N. A. Spaldin, and F. Nolting, Magnetoelectroelastic control of magnetism in an artificial multiferroic, Phys. Rev B 94, 014401 (2016).

[31] J. J. Yang, Y. G. Zhao, H. F. Tian, L. B. Luo, H. Y. Zhang, Y. J. He, and H. S. Luo, Electric field manipulation of magnetization at room temperature in multiferroic $\mathrm{CoFe}_{2} \mathrm{O}_{4} / \mathrm{Pb}\left(\mathrm{Mg}_{1 / 3} \mathrm{Nb}_{2 / 3}\right)_{0.7} \mathrm{Ti}_{0.3} \mathrm{O}_{3}$ heterostructures, Appl. Phys. Lett. 94, 212504 (2009).
[32] I. Fina, A. Quintana, X. Martí, F. Sánchez, M. Foerster, L. Aballe, J. Sort, and J. Fontcuberta, Reversible and magnetically unassisted voltage-driven switching of magnetization in FeRh/PMN-PT, Appl. Phys. Lett. 113, 152901 (2018).

[33] I. Fina, A. Quintana, J. Padilla-Pantoja, X. Marti, F. Macia, F. Sanchez, M. Foerster, L. Aballe, J. Fontcuberta, and J. Sort, Electric-field-adjustable time-dependent magnetoelectric response in martensitic FeRh alloy, ACS Appl. Mater. Interfaces 9, 15577 (2017).

[34] L. Shu, Z. Li, J. Ma, Y. Gao, L. Gu, Y. Shen, Y. Lin, and C. W. Nan, Thickness-Dependent voltage-modulated magnetism in multiferroic heterostructures, Appl. Phys. Lett. 100, 022405 (2012).

[35] U. Bauer, L. Yao, A. J. Tan, P. Agrawal, S. Emori, H. L. Tuller, S. van Dijken, and G. S. D. Beach, Magnetoionic control of interfacial magnetism, Nat. Mater. 14, 174 (2015).

[36] D. A. Gilbert, A. J. Grutter, E. Arenholz, K. Liu, B. J. Kirby, J. A. Borchers, and B. B. Maranville, Structural and magnetic depth profiles of magneto-ionic heterostructures beyond the interface limit, Nat. Commun. 7, 12264 (2016).

[37] L. Zhang, S. Zeng, X. Yin, T. C. Asmara, P. Yang, K. Han, Y. Cao, W. Zhou, D. Wan, C. S. Tang, A. Rusydi, Ariando, and T. Venkatesan, The mechanism of electrolyte gating on high- $\mathrm{T}_{\mathrm{C}}$ cuprates: the role of oxygen migration and electrostatics, ACS Nano 11, 9950 (2017).

[38] Q. Zhang, X. He, J. Shi, N. Lu, H. Li, Q. Yu, Z. Zhang, L.-Q. Chen, B. Morris, Q. Xu, P. Yu, L. Gu, K. Jin, and C.W. Nan, Atomic-resolution imaging of electrically induced oxygen vacancy migration and phase transformation in $\mathrm{SrCoO}_{2.5-\sigma}$, Nat. Commun. 8, 104 (2017).

[39] A. Quintana, E. Menéndez, E. Isarain-Chávez, J. Fornell, P. Solsona, F. Fauth, M. D. Baró, J. Nogués, E. Pellicer, and J. Sort, Tunable magnetism in nanoporous $\mathrm{CuNi}$ alloys by reversible voltage-driven element-selective redox processes, Small 4, 1704396 (2018).

[40] A. Quintana, E. Menéndez, M. O. Liedke, M. Butterling, A. Wagner, V. Sireus, P. Torruella, S. Estradé, F. Peiró, J. Dendooven, C. Detavernier, P. D. Murray, D. A. Gilbert, K. Liu, E. Pellicer, J. Nogués, and J. Sort, Voltage-controlled $\mathrm{ON}-\mathrm{OFF}$ ferromagnetism at room temperature in a single metal oxide film, ACS Nano 12, 10291 (2018).

[41] C. Bi, Y. Liu, T. Newhouse-Illige, M. Xu, M. Rosales, J. W. Freeland, O. Mryasov, S. Zhang, S. G. E. te Velthuis, and W. G. Wang, Reversible Control of Co Magnetism by Voltage-Induced Oxidation, Phys. Rev. Lett. 113, 267202 (2014).

[42] N. Mehmood, R. S. Turtelli, R. Grössinger, and M. Kriegisch, Magnetostriction of polycrystalline $\mathrm{Fe}_{100-\mathrm{x}} \mathrm{Al}_{\mathrm{x}}$ ( $\mathrm{x}=15,19,25)$, J. Alloys Compd. 322, 1609 (2010).

[43] R. Grössinger, R. S. Turtelli, and N. Mehmood, Materials with high magnetostriction, IOP Conf. Ser.: Mater. Sci. Eng. 60, 012002 (2014).

[44] Y. Wei, G. Yang, H. Yang, and S. Xi, Selective control of magnetism in FeAl/PIN-PMN-PT using an electric field, Appl. Phys. Lett. 114, 082404 (2019).

[45] J. H. Westbrook and R. L. Fleischer, Intermetallic Compounds, Structural applications of intermetallic compounds, (John Wiley \& Sons, New York, 2000), 2nd ed., Vol. 3. 
[46] P. Weaver and M. G. Cain, Chapter: Temperature dependence of ferroelectric and piezoelectric properties of PZT ceramics, in Characterisation of ferroelectric bulk materials and thin films, edited by M. G. Cain (Springer, 2014). Series in Measurement Science and Technology, Vol. 2.

[47] H. Ouchi, K. Nagano, and S. Hayakawa, Materials with high Magnetostriction, J. Am. Ceram. Soc. 48, 630 (1965).

[48] H. Ouchi, Piezoelectric properties and phase relations of $\mathrm{Pb}\left(\mathrm{Mg}_{1 / 3} \mathrm{Nb}_{2 / 3}\right) \mathrm{O}_{3}-\mathrm{PbTiO}_{3}-\mathrm{PbZrO}_{3}$ ceramics with Barium or Strontium substitutions, J. Am. Ceram. Soc. 51, 169 (1968).

[49] A. Biancoli, C. M. Fancher, J. L. Jones, and D. Damjanovic, Breaking of macroscopic centric symmetry in paraelectric phases of ferroelectric materials and implications for Flexoelectricity, Nat. Mater. 14, 224 (2015).

[50] M. Foerster, I. Fina, S. Finizio, B. Casals, A. Mandziak, F. Fauth, and L. Aballe, Disclosing odd symmetry, strain driven magnetic response of Co on Pt/PMN-PT ( $\left.\begin{array}{lll}0 & 1 & 1\end{array}\right)$, J. Phys.: Condens. Matter. 31, 084003 (2019).

[51] See Supplemental Material at http://link.aps.org/supplem ental/10.1103/PhysRevApplied.12.014041 for further structural, compositional, magnetic and magnetoelectric characterization.
[52] M. Zhernenkov, M. R. Fitzsimmons, J. Chlistunoff, J. Majewski, I. Tudosa, and E. E. Fullerton, Electric-field modification of magnetism in a thin CoPd film, Phys. Rev. B 82, 024420 (2010).

[53] T. Zhao, A. Scholl, F. Zavaliche, K. Lee, M. Barry, A. Doran, M. P. Cruz, Y. H. Chu, C. Ederer, N. A. Spaldin, R. R. Das, D. M. Kim, S. H. Baek, C. B. Eom, and R. Ramesh, Electrical control of antiferromagnetic domains in multiferroic $\mathrm{BiFeO}_{3}$ films at room temperature, Nat. Mater. 5, 823 (2006).

[54] Y. Wang, C. Nelson, A. Melville, B. Winchester, S. Shang, Z.-K. Liu, D. G. Schlom, X. Pan, and L.Q. Chen, $\mathrm{BiFeO}_{3}$ domain wall energies and structures: a combined experimental and density functional theory $+\mathrm{U}$ study, Phys. Rev. B 110, 267601 (2013).

[55] P. Marton, I. Rychetsky, and J. Hlinka, Domain walls of ferroelectric $\mathrm{BaTiO}_{3}$ within the Ginzburg-Landau-Devonshire phenomenological model, Phys. Rev. B 81, 144125 (2010).

[56] Z. Gareeva, O. Diéguez, J. Íñiguez, and A. K. Zvezdin, Complex domain walls in $\mathrm{BiFeO}_{3}$, Phys. Rev. B 91, 060404(R) (2015). 